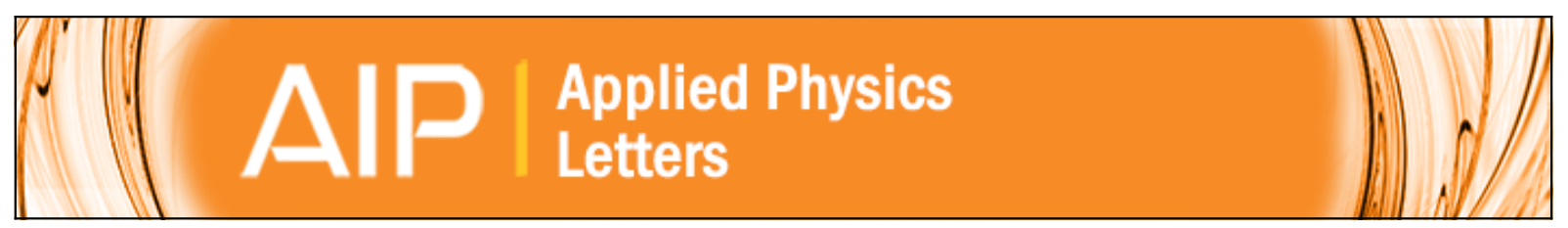

\title{
Bond formation of surface-tethered receptor-ligand pairs in relative separation
}

Jin Qian, Yuan Lin, Hongyuan Jiang, and Haimin Yao

Citation: Applied Physics Letters 103, 223702 (2013); doi: 10.1063/1.4834915

View online: http://dx.doi.org/10.1063/1.4834915

View Table of Contents: http://scitation.aip.org/content/aip/journal/apl/103/22?ver=pdfcov

Published by the AIP Publishing

\section{Articles you may be interested in}

Effect of ligand diffusion on occupancy fluctuations of cell-surface receptors

J. Chem. Phys. 139, 121910 (2013); 10.1063/1.4816105

Kinetics of receptor occupancy during morphogen gradient formation

J. Chem. Phys. 138, 244105 (2013); 10.1063/1.4811654

Spinodal decomposition and the emergence of dissipative transient periodic spatio-temporal patterns in acentrosomal microtubule multitudes of different morphology

Chaos 23, 023120 (2013); 10.1063/1.4807909

The diffusive finite state projection algorithm for efficient simulation of the stochastic reaction-diffusion master equation

J. Chem. Phys. 132, 074101 (2010); 10.1063/1.3310809

Anomalous reaction-diffusion as a model of nonexponential DNA escape kinetics

J. Chem. Phys. 132, 025103 (2010); 10.1063/1.3290987

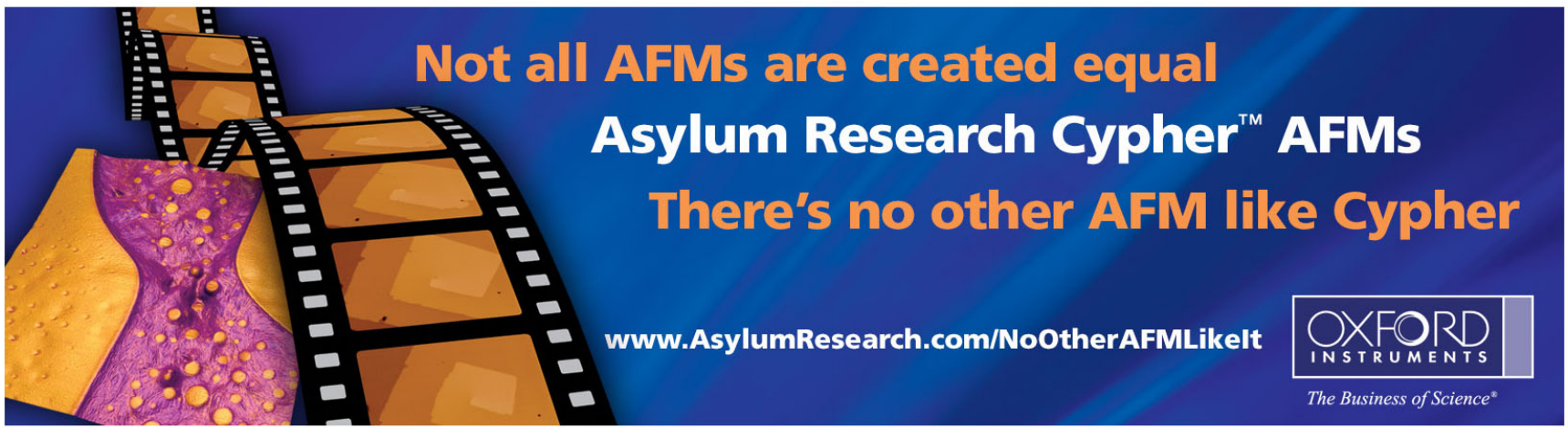




\title{
Bond formation of surface-tethered receptor-ligand pairs in relative separation
}

\author{
Jin Qian, ${ }^{1,2, a)}$ Yuan Lin, ${ }^{3}$ Hongyuan Jiang, ${ }^{4}$ and Haimin Yao $^{2,5, a)}$ \\ ${ }^{1}$ Department of Engineering Mechanics, Zhejiang University, Hangzhou, Zhejiang 310027, China \\ ${ }^{2}$ Department of Mechanical Engineering, The Hong Kong Polytechnic University, Hong Kong, China \\ ${ }^{3}$ Department of Mechanical Engineering, The University of Hong Kong, Hong Kong, China \\ ${ }^{4}$ Department of Modern Mechanics, The University of Science and Technology of China, Hefei, \\ Anhui 230026, China \\ ${ }^{5}$ The Hong Kong Polytechnic University Shenzhen Research Institute, Shenzhen 518057, China
}

(Received 31 August 2013; accepted 13 November 2013; published online 26 November 2013)

\begin{abstract}
We theoretically and numerically investigate the interplay between diffusion of a surface-bound receptor and its reaction with an opposing ligand. Special attention has been paid to the mechanical regulation of bond association by varying the initial gap distance and relative separation speed between the protein-bearing surfaces. Such diffusion-reaction coupling effects can cause the apparent on-rate or reciprocal of the average waiting time for bond formation, to be not constant, but instead a function sensitive to the system parameters that affect the transport of proteins. The results provide a quantitative understanding of how significantly the transport mechanism can affect overall binding behavior of molecular interactions and call for a paradigm shift in modeling receptor-ligand bond association when the protein-bearing surfaces are in relative separation. (C) 2013 AIP Publishing LLC. [http://dx.doi.org/10.1063/1.4834915]
\end{abstract}

Reversible binding and unbinding between specific receptors and ligands mediate a variety of adhesion-based processes in cellular behaviors such as spreading, migration, differentiation, and polarization. ${ }^{1}$ Binding and unbinding of receptor-ligand bonds are commonly characterized by measuring their association and dissociation kinetic rates, which are tightly regulated by physical factors and biochemical signals that are still being elucidated. The force dependence of bond dissociation has been extensively studied at singlemolecule level by force-clamp ${ }^{2-4}$ or force-ramp ${ }^{5-7}$ spectroscopy, which fosters Kramers' theory of thermally assisted escape crossing transitional energy barrier. ${ }^{8}$ In contrast, experimental quantification of bond association kinetics is few and its detailed regulation mechanisms remain to be fully understood from theoretical point of view. ${ }^{9,10}$

The intrinsic association rate between complementary receptor-ligand molecules is typically studied in threedimensional situations where at least one protein is in solution. ${ }^{11}$ However, cell adhesion often takes place between opposing surfaces of cell membrane or extracellular matrix, where specific receptor and ligand molecules are anchored. Therefore, modeling the association dynamics between surface-tethered molecular pairs is more physiologically relevant. The association of receptor-ligand bonds has been shown to play a crucial role in understanding the collective behaviors of multi-bond clusters, where enhanced strength and stability are attributed by bond reforming among the bond ensemble. ${ }^{12-14}$ The failure of multiple catch-bonds at various loading conditions has also been studied, where the bond association is modeled by a rate expression in closed form that decays with increased receptor-ligand distance. ${ }^{15}$ For more multi-bond adhesion models, receptor-ligand association has been considered as similar rate process in studying cells'

a)Electronic addresses: jqian@zju.edu.cn and mmhyao@polyu.edu.hk. spreading on ${ }^{16}$ and detaching from underlying substrates, ${ }^{17}$ coherently with other important aspects such as nonspecific interaction, dissociation of specific bonds, in-membrane diffusion of receptors, as well as membrane deformation. Recently, the cooperative adhesion of multi-bond clusters has also been investigated under a dynamic loading condition, both theoretically and experimentally, where the opposing protein-bearing surfaces are separated at a constant speed. ${ }^{18-20}$

Most of the existing studies ${ }^{12-20}$ have assumed that the rate of bond formation is constant or dependent on the receptor-ligand distance, with few concerns about the relative motion in affecting the binding process. However, relative motion between protein-bearing surfaces is inevitable in many scenarios of cell adhesion. In the cases of dynamic membrane peeling, ${ }^{21}$ filopodia protrusion, ${ }^{22}$ or cell rolling on surfaces, ${ }^{23}$ the reacting protein molecules are bound on cell or matrix surfaces, and the intra- or extra-cellular environments, e.g., actin contractility or external flow, drive the motion of protein-carrying surfaces, resulted in bond formation against dynamic separation. We should pointed out that Chang and Hammer ${ }^{24}$ have brilliantly conceived a quantitative approach for the association rate of surface-tethered molecules when the surfaces are sliding parallel to each other, but a model of bond formation is still lacking to account for the effects of relative separation between the opposing protein-bearing surfaces.

Here, we present a theoretical model to understand the association kinetics of surface-bound protein pairs, with special attention paid to the mechanical regulation of bond association by varying the initial gap distance and relative separation speed between the specific proteins. The work should be of immediate use in understanding the type of cell adhesion problems in Refs. 18-20, where the transport of reactive biomolecules and surface separation are competing factors in bond formation. In our modeling, the essential 
feature of receptor-ligand association process is made complicated by the fact that the adhesion between receptor and ligand molecules requires two essential steps: it first requires a transport-governed phase for the opposing reactants to be close in contact, overcoming the initial gap distance and incremental surface separation, then biochemical reaction can take effect at the intrinsic rate constant. Thus, the apparent kinetic information from the process is really mixed time scales combining both the transport and reaction terms. In single pairs of receptor and ligand molecules residing at cell-cell or cellmatrix interface, transport of proteins can be a limiting factor of the entire association process and the rate of bond formation is expected to be affected by the initial gap distance, relative separation speed, medium viscosity, stiffness of proteins, etc., that can possibly influence the transport process.

Consider a biophysical model describing the transportreaction process of a receptor-ligand pair (Figure 1(a), for example), where two reactive biomolecules are tethered to opposing surfaces with initial distance $d_{0}$ and separated at a constant speed $v \geq 0$. The current surface separation at time $t$ is simply $d=d_{0}+v t$. We postulate that the instantaneous motion of the receptor against the tethering is essentially one dimensional. Suppose that the pair of receptor and ligand can bind at an intrinsic constant rate $k_{\mathrm{on}}^{0}$ when they fall into a binding distance $l_{\text {bind }}$ in close proximity; when they are apart beyond the binding distance, the receptor has to first approach the binding pocket of the ligand through Brownian motion. We further assume for simplicity that the ligand is fixed on one surface while the receptor is tethered to the other by a linear spring with stiffness $k_{\mathrm{b}}$ and rest length $l_{\mathrm{b}}$ (Figure 1(b)). A large ensemble of nominally identical trials is described by means of the probability density function $\rho(x, t)$, i.e., the probability of finding the receptor in position $x$ at time $t$. We denote the flux of receptor motion in $x$ direction at time $t$ by $j(x, t)$ and the local bond association rate by $s(x, t)$.

(a)
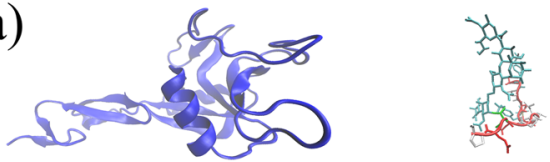

(b)

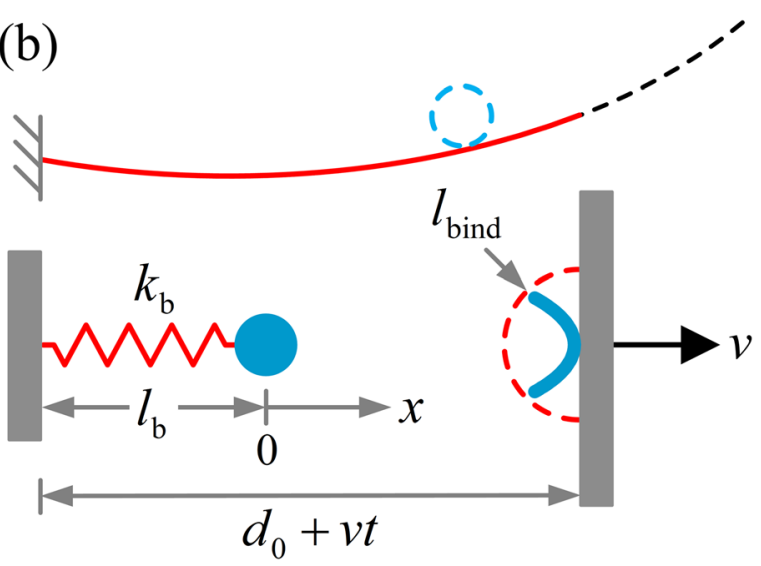

FIG. 1. (a) Bond association between opposing receptor and ligand molecules. The P-selectin (Protein Data Bank code: 1G1Q) and PSGL-1 (Protein Data Bank code: 1G1S) molecules are used as an example. (b) The process of bond association is modeled as two essential steps: diffusion of receptor molecules along the energy landscape of a truncated harmonic potential and bond formation between the opposing molecules in close proximity.
Following the conservation law, the local rate of change in $\rho(x, t)$ must be equal to the negative of the local flux divergence, minus the local loss due to bond association, or

$$
\frac{\partial \rho(x, t)}{\partial t}=-\frac{\partial j(x, t)}{\partial x}-s(x, t)
$$

throughout $-l_{\mathrm{b}} \leq x \leq d_{0}+v t-l_{\mathrm{b}}$ in the coordinate setting, $x=0$ picked as the equilibrium position of the tethering spring (Figure 1(b)). With the understanding that the flux of receptor motion should be proportional to the local gradient in the chemical potential, $j(x, t)$ can be related to the probability density function $\rho(x, t)$ and the landscape of potential energy $U(x)$ through $^{25}$

$$
j(x, t)=-D\left(\frac{\partial \rho(x, t)}{\partial x}+\frac{\rho(x, t)}{k_{\mathrm{B}} T} \frac{\mathrm{d} U(x)}{\mathrm{d} x}\right),
$$

where

$$
U(x)=\frac{k_{\mathrm{b}} x^{2}}{2},\left(-l_{\mathrm{b}} \leq x \leq d_{0}+v t-l_{\mathrm{b}}\right)
$$

is the truncated harmonic potential due to the tethering, $k_{\mathrm{b}}$ being the spring constant, $k_{\mathrm{B}}$ in Eq. (2) is the Boltzmann constant, $T$ is absolute temperature, and $D$ is the diffusivity of receptor molecules, which is a function of receptor size and medium viscosity by Stokes-Einstein relation. The reaction term in Eq. (1) is

$$
\begin{aligned}
s(x, t)= & k_{\text {on }}^{0} \rho(x, t)\left(H\left(x-\left(d_{0}+v t-l_{\mathrm{b}}-l_{\text {bind }}\right)\right)\right. \\
& \left.-H\left(x-\left(d_{0}+v t-l_{\mathrm{b}}\right)\right)\right),
\end{aligned}
$$

according to the first-order kinetic equation. Regarding the depleting reaction term $s(x, t)$ via receptor-ligand bond formation, there is no basis to expect association occurrence when the opposing molecules are far from each other, and the region where reaction can possibly happen is therefore characterized through the length scale $l_{\text {bind }}$, as defined by the reaction window $H\left(x-\left(d_{0}+v t-l_{\mathrm{b}}-l_{\text {bind }}\right)\right)-H\left(x-\left(d_{0}+v t-l_{\mathrm{b}}\right)\right)$ in Eq. (4), $H$ being the Heaviside step function. Combination of Eqs. (1)-(4) leads to the Smoluchowski type partial differential equation governing the spatiotemporal evolution of the probability density function $\rho(x, t)$ :

$$
\begin{aligned}
& \frac{\partial \rho(x, t)}{\partial t}-D \frac{\partial}{\partial x}\left(\frac{\partial \rho(x, t)}{\partial x}+\frac{k_{\mathrm{b}} x}{k_{\mathrm{B}} T} \rho(x, t)\right) \\
& \quad+k_{\mathrm{on}}^{0} \rho(x, t)\left(H\left(x-\left(d_{0}+v t-l_{\mathrm{b}}-l_{\mathrm{bind}}\right)\right)\right. \\
& \left.\quad-H\left(x-\left(d_{0}+v t-l_{\mathrm{b}}\right)\right)\right)=0,
\end{aligned}
$$

which is subjected to the moving boundary condition $j\left(-l_{\mathrm{b}}, t\right)=j\left(d_{0}+v t-l_{\mathrm{b}}, t\right)=0$ indicating that the surfaces are impenetrable, and initial condition $\rho(x, 0)=\delta(x)$, where $\delta(x)$ is Dirac delta function. When the reaction term is absent, the model immediately reduces to the classical Smoluchowski equation describing Brownian motion in a harmonic potential.

Choosing $l_{\mathrm{b}}$ and $1 / k_{\mathrm{on}}^{0}$ as the basic length and time scales in the problem, we proceed by grouping all the system parameters as follows: 

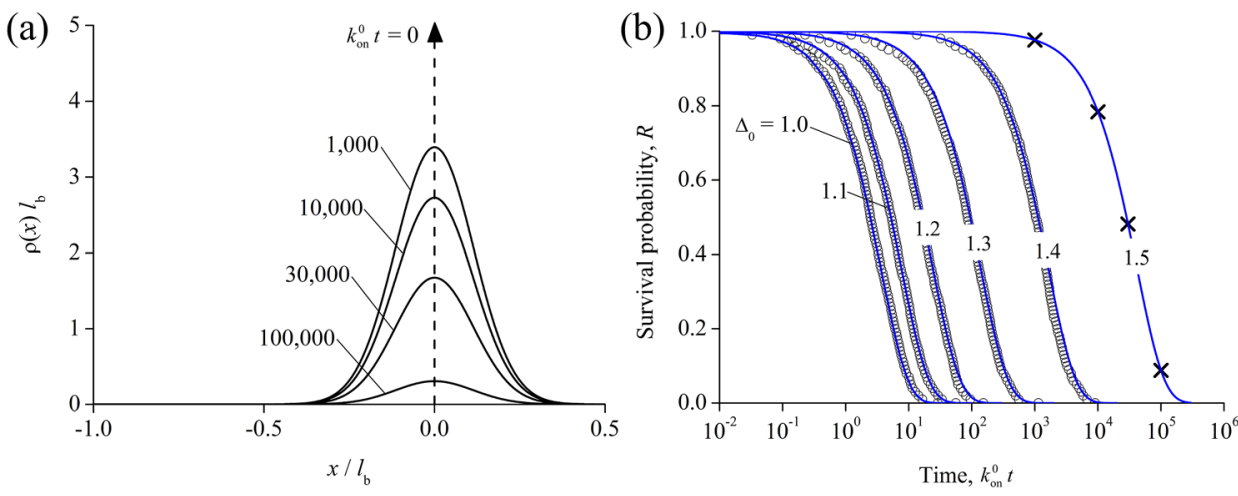

FIG. 2. (a) Time evolution of the probable location of unbound receptor molecules $\left(\Delta_{0}=1.5\right)$. (b) Plots of the survival probability $R$, defined as the probability that a single receptorligand pair remains unbound versus (normalized) time $k_{\mathrm{on}}^{0} t$ for a range of initial gap distance $\Delta_{0}$. Model predictions (curves) and Monte Carlo simulations (circles) agree well to each other. Crosses represent the certain time points appearing in (a).

$$
\lambda=\frac{D}{k_{\mathrm{on}}^{0} l_{\mathrm{b}}^{2}}, \quad \kappa=\frac{k_{\mathrm{b}} l_{\mathrm{b}}^{2}}{k_{\mathrm{B}} T}, \quad L_{\text {bind }}=\frac{l_{\text {bind }}}{l_{\mathrm{b}}}, \quad \Delta_{0}=\frac{d_{0}}{l_{\mathrm{b}}}, \quad V=\frac{v}{k_{\mathrm{on}}^{0} l_{\mathrm{b}}} .
$$

According to the representative values $l_{\mathrm{b}}=25 \mathrm{~nm},{ }^{26,27}$ $k_{\mathrm{b}}=0.5 \mathrm{pN} / \mathrm{nm},{ }^{26,27}$ and $l_{\text {bind }}=1 \mathrm{~nm}$ (Ref. 28) for integrin molecules, we estimate that $\kappa=76$ and $L_{\text {bind }}=0.04$. The diffusivity $D$ of receptor molecules is expected to be $\sim 4 \times 10^{-11} \mathrm{~m}^{2} / \mathrm{s}$ from Stokes-Einstein relation with typical values of receptor radius ( $\sim 5 \mathrm{~nm}$, Ref. 29) and medium viscosity ( $\sim 1 \mathrm{mPa} \cdot \mathrm{s}$, Ref. 30$)$. Taking $k_{\text {on }}^{0}$ to be $\sim 6 \times 10^{4} / \mathrm{s},{ }^{31} \lambda$ is estimated to be a quantity about unity. We then vary the rest 2 parameters in Eq. (6) to examine the dependence of apparent association dynamics on the initial gap distance and relative separation speed in the competition between physical transport and biochemical reaction, as explicitly described by Eq. (5) and associated boundary/initial conditions.

The solution to the boundary value problem has been carried out by numerical finite element method. The numerical procedure leads to discrete values of the probability distribution $\rho(x, t)$ at 1000 equally spaced values of $x$ at incremental time step $\delta$, accounting for the dynamic evolution in $\rho(x, t)$ and the moving boundary. Within an ensemble of nominally identical trials, the association dynamics is understood by calculating the fraction of all events, which remains unbound after total elapsed time $t$ in the observation, denoted as $R(t)$. Equivalently, $R(t)$ represents the probability that a single receptor-ligand pair remains unbound after time $t$, termed as "survival probability" in the following discussions.
Let us first examine the behavior of bond formation when the opposing surfaces are stationary to each other (i.e., $V=0)$. Clearly, $R(t)$ is the area under each distribution of $\rho(x, t)$ at given time points, as demonstrated in Figure 2(a). This quantity generally has the initial value $R(0)=1$, exhibits the asymptotic behavior $R(t) \rightarrow 0$ as time becomes sufficiently large (Figure 2(b)), and its dynamic behavior is controlled by the coupling between intrinsic bond association kinetics and the underlying diffusion process of receptor molecules. The theoretical approach is validated by its excellent agreement with the corresponding Monte Carlo simulations. ${ }^{32}$

The average waiting time (dimensionless) for bond association, or reciprocal of the apparent on-rate, is therefore

$$
T_{\mathrm{w}}=k_{\mathrm{on}}^{0} \int_{0}^{\infty}-\dot{R}(t) \times t \mathrm{~d} t
$$

from the $R$ versus $t$ relation given in Figure 2(b). The dependence of $T_{\mathrm{w}}$ on $\Delta_{0}$ is plotted in Figure 3(a), favorably compared to the mean first passage time of bond making from the Monte Carlo simulations. ${ }^{32}$

In the case that $V=0$ and $\lambda=D /\left(k_{\mathrm{on}}^{0} l_{\mathrm{b}}^{2}\right)$ is sufficiently large, the transport of a receptor molecule in its configuration state is much faster than its reaction with ligand, so that $\rho(x, t)$ should obey the Boltzmann distribution at any time

$$
p(x)=\frac{1}{Z} \exp \left(-\frac{k_{\mathrm{b}} x^{2}}{2 k_{\mathrm{B}} T}\right)
$$
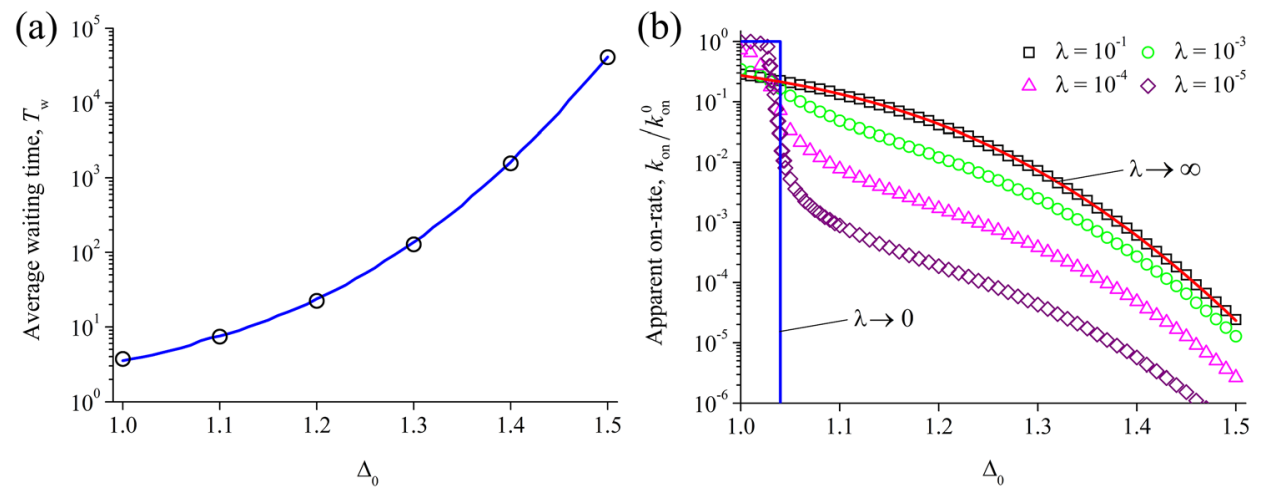

FIG. 3. (a) The average waiting time $T_{\mathrm{w}}$ as a function of the gap distance $\Delta_{0}$ between the opposing surfaces when $V=0$ (Curve: model predictions; Symbols: Monte Carlo simulations). (b) The apparent on-rate $k_{\mathrm{on}} / k_{\mathrm{on}}^{0}$, defined as the reciprocal of the average waiting time $T_{\mathrm{w}}$, as a function of the surface gap distance $\Delta_{0}$. The results show a smooth transition between fast and slow molecule diffusion relative to the biochemical reaction, governed by the dimensionless parameter $\lambda=D /\left(k_{\mathrm{on}}^{0} l_{\mathrm{b}}^{2}\right)$. The on-rate results based on Boltzmann distribution $(\lambda \rightarrow \infty)$ in Refs. 31 and 33 and "frozen" receptors $(\lambda \rightarrow 0)$ serve as the two limiting cases. 

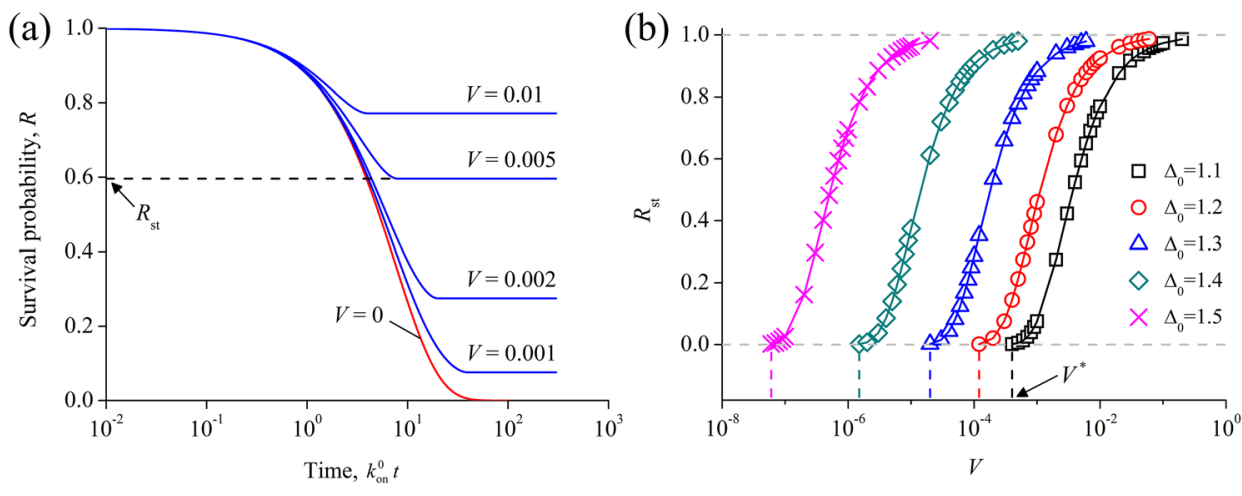

FIG. 4. (a) Time-varying behaviors of the survival probability $R$ when $V>0$ $\left(\Delta_{0}=1.1\right)$. $R$ first decreases from its initial value of unity and reaches a nonzero plateau value, denoted as $R_{\text {st }}$, as $V$ is increased to beyond a critical value (i.e., $\left.V^{*}\right)$. (b) $R_{\text {st }}$, representing the probability that a single receptor-ligand pair remains unbound for infinitely long time, as a function of the separation speed $V . V^{*}$ can therefore be determined as the critical speed that leads to non-zero fraction of "hopeless" bond formation. where $Z$ is the partition sum that can be explicitly determined by the normalization condition $\int_{-l_{\mathrm{b}}}^{d_{0}-l_{\mathrm{b}}} p(x) \mathrm{d} x=1 .^{31,33}$ The apparent association rate $k_{\mathrm{on}}$ is therefore $k_{\mathrm{on}}^{0}$ multiplied by the probability of the receptor falling into the binding region $\left(d_{0}-l_{\mathrm{b}}-l_{\text {bind }}, d_{0}-l_{\mathrm{b}}\right)$, which recovers the on-rate description adopted in Refs. 31 and 33 as

$$
k_{\mathrm{on}}=\frac{k_{\mathrm{on}}^{0}}{Z} \int_{d_{0}-l_{\mathrm{b}}-l_{\text {bind }}}^{d_{0}-l_{\mathrm{b}}} \exp \left(-\frac{k_{\mathrm{b}} x^{2}}{2 k_{\mathrm{B}} T}\right) \mathrm{d} x .
$$

In the opposite limit $\lambda \rightarrow 0$, the transport of receptor is nearly "frozen" compared to the time scale of bond reaction. As such, the apparent on-rate $\left(k_{\mathrm{on}} / k_{\mathrm{on}}^{0}\right)$ will be unity if $\Delta_{0} \leq 1.04$, where the receptor and ligand molecules are already in contact or negligibly small if $\Delta_{0}>1.04$ because the transport process takes too much time. Figure 3(b) plots the apparent on-rate as a function of the surface separation $\Delta_{0}$ for several values of $\lambda$, indicating a smooth transition between fast and slow molecule diffusion relative to biochemical reaction. Given $V=0$, the gap distance $\Delta_{0}$ governs the relative contribution from transport and reaction terms to the overall binding process, and roughly speaking, the boundary between reaction- and transport-limited regions is at $d_{0}^{*}=l_{\mathrm{b}}+l_{\text {bind }}$. Following the approach by Ref. 34 , the dominating "resistance" to bond association is from reaction for $d_{0}<d_{0}^{*}$, or from transport for $d_{0}>d_{0}^{*}$. Variations in surface gap distance, molecule length, binding distance, as well as tethering stiffness can result in a broad range of kinetic association response that may relate to tight regulation mechanisms in cell adhesion.

To further examine the transport-reaction coupling in the presence of receptor-ligand separation (i.e., $V>0$ ), the values of $R(t)$ at any particular time points are also extracted by numerical integration of the probability distribution over those spatial intervals within $-l_{\mathrm{b}} \leq x \leq d_{0}+v t-l_{\mathrm{b}}$ for various values of $V$ (i.e., $\left.v /\left(k_{\mathrm{on}}^{0} l_{\mathrm{b}}\right)\right)$, as shown in Figure 4(a). Interestingly, as $V$ increases to above a critical value, say $V^{*}$, $R(t)$ first decreases from its initial value of unity until a nonzero steady-state value is reached, denoted by $R_{\text {st }}$ in Figure 4(a). In other words, $R_{\mathrm{st}}$ represents the certain fraction of total events for which bond association never happens or equivalently the probability that one single bond will remain unbound for infinitely long time. Clearly, the critical separation speed $V^{*}$ that leads to non-zero $R_{\text {st }}$ should drop down as the initial gap distance $\Delta_{0}$ increases (Figure 4(b)). These results call for a paradigm shift in modeling the receptorligand bond association when the protein-bearing surfaces are in relative separation: ${ }^{18-20}$ depending on the separation speed $V$, the rate of receptor-ligand association should take negligible value for the probability of $R_{\text {st }}$, or follow certain distributions for probability $\left(1-R_{\mathrm{st}}\right)$ as the portion of curves before $R(t)=R_{\mathrm{st}}$ is reached, referring to Figure 4(a).

The augment of association waiting time to infinity by threshold values of separation speed, as demonstrated in Figures 4(a) and 4(b), suggests an appealing interpretation: the overall rate of bond formation is governed by two individual "resistance" in series: receptor diffusion into the reaction region in proximity to the opposing ligand and intrinsic reaction between the biomolecules. Compared to the case where protein-bearing surfaces are stationary to each other, the separation speed $V$ appears as a factor adding more barriers to the former process. As $V$ is smaller than $V^{*}$, the opposing protein pair is in relative separation but receptor transport is relatively fast, enabling the receptor to catch up with the moving ligand for successful bond making; As $V$ goes beyond $V^{*}$, the receptor and ligand are separated too fast so that it is hardly possible for the receptor to get into the reaction region irrespective of time.

As a relevant and important application of our work in biological functions, the immunological binding between $\mathrm{T}$ cell receptors (TCRs) on T cell surfaces and peptide-major histocompatibility complex (MHC) molecules on antigen-presenting cells (APCs) has been revealed to strongly depend on the gap distance between the two surfaces: ${ }^{35} \mathrm{~T}$ cell and APC membranes at $40 \mathrm{~nm}$ apart have low binding affinity, while formation of TCR-peptide-MHC bonds is greatly promoted when the membrane spacing is reduced to $\sim 15 \mathrm{~nm}$ by actin-based protrusion. According to the present analysis, it is reasonable to speculate that $15 \mathrm{~nm}$ is a length scale close to the combined length of molecules and binding distance $\left(d_{0}^{*}\right)$, so that positioning protein anchoring surfaces at this distance apart enables the opposing molecules to find each other for bond making. Furthermore, the change of membrane spacing is achieved by retraction or protrusion of actin-based structures, where dynamic surface separation or approach is inevitable. The present framework and results should be helpful in understanding the actual association kinetics in such physiological conditions.

In summary, we have performed both theoretical and numerical analyses to investigate the coupling process of physical transport and biochemical reaction that mediate receptor-ligand association. Controlled initial gap distance and relative separation speed, together with other involved physical parameters including receptor diffusivity, molecular length, binding distance, tethering stiffness, etc., are found to 
govern the transport- and reaction-limited transition in bond formation. The results should be useful in understanding phenomena such as dynamic membrane peeling, actin-driven membrane separation, and cell rolling on surfaces, where bond association occurs in a scenario of relative surface separation. We have also connected the apparent waiting time of bond formation to the intrinsic association rate constant, so the proposed model can be used in an inverse approach to determine intrinsic kinetic parameters from apparent kinetic measurements on bond association. It should be pointed out that the present study is based on one-dimensional configuration, which may be extended to higher dimensions in more sophisticated models. To achieve that, the tethered diffusion process of protein molecules must be considered in a threedimensional energy landscape, which is unbounded in the two directions parallel to the protein-bearing surfaces but limited by impenetrable walls in the perpendicular direction. Furthermore, the binding radius should set a hemi-spherical zone that captures the molecules in the three-dimensional landscape. These factors will involve complicated mathematical or geometrical considerations upon the present onedimensional approach.

This work was supported by the "Thousand Young Talents Program" of China, the National Natural Science Foundation of China (Nos. 11202184, 11321202, and 11072273), and the Fundamental Research Funds for Central Universities (No. 2012QNA4023) of China. Partial support from the Departmental General Research Funds (No. 4ZZA8) of the Hong Kong Polytechnic University is also acknowledged.

${ }^{1}$ B. Alberts, A. Johnson, J. Lewis, M. Raff, K. Roberts, and P. Walter, Molecular Biology of the Cell (Garland Science, New York, 2002).

${ }^{2}$ B. T. Marshall, M. Long, J. W. Piper, T. Yago, R. P. McEver, and C. Zhu, Nature 423(6936), 190 (2003).

${ }^{3}$ J. Z. Lou, T. Yago, A. G. Klopocki, P. Mehta, W. Chen, V. I. Zarnitsyna, N. V. Bovin, C. Zhu, and R. P. McEver, J. Cell Biol. 174(7), 1107 (2006).

${ }^{4}$ K. K. Sarangapani, J. Qian, W. Chen, V. I. Zarnitsyna, P. Mehta, T. Yago, R. P. McEver, and C. Zhu, J. Biol. Chem. 286(37), 32749 (2011).
${ }^{5}$ E. Evans and K. Ritchie, Biophys. J. 72(4), 1541 (1997).

${ }^{6}$ G. Hummer and A. Szabo, Biophys. J. 85(1), 5 (2003).

${ }^{7}$ J. Kim, C. Z. Zhang, X. H. Zhang, and T. A. Springer, Nature 466(7309), 992 (2010).

${ }^{8}$ P. Hanggi, P. Talkner, and M. Borkovec, Rev. Mod. Phys. 62(2), 251 (1990).

${ }^{9}$ T. Yago, V. I. Zarnitsyna, A. G. Klopocki, R. P. McEver, and C. Zhu, Biophys. J. 92(1), 330 (2007).

${ }^{10}$ W. Chen, E. A. Evans, R. P. McEver, and C. Zhu, Biophys. J. 94(2), 694 (2008).

${ }^{11}$ G. I. Bell, Science 200(4342), 618 (1978).

${ }^{12}$ T. Erdmann and U. S. Schwarz, Phys. Rev. Lett. 92(10), 108102 (2004).

${ }^{13}$ J. Qian, J. Z. Wang, Y. Lin, and H. J. Gao, Biophys. J. 97(9), 2438 (2009).

${ }^{14}$ J. Qian and H. J. Gao, Plos One 5(8), e12342 (2010).

${ }^{15}$ L. Sun, Q. H. Cheng, H. J. Gao, and Y. W. Zhang, J. R. Soc., Interface 9(70), 928 (2012).

${ }^{16}$ L. Sun, Q. H. Cheng, H. J. Gao, and Y. W. Zhang, Phys. Rev. E 79(6), 061907 (2009).

${ }^{17}$ Q. H. Cheng, P. Liu, H. J. Gao, and Y. W. Zhang, J. Mech. Phys. Solids 57(2), 205 (2009).

${ }^{18}$ U. Seifert, Phys. Rev. Lett. 84(12), 2750 (2000).

${ }^{19}$ T. Erdmann and U. S. Schwarz, Europhys. Lett. 66(4), 603 (2004).

${ }^{20}$ T. Erdmann, S. Pierrat, P. Nassoy, and U. S. Schwarz, Europhys. Lett. 81, 48001 (2008).

${ }^{21}$ M. D. Ward, M. Dembo, and D. A. Hammer, Biophys. J. 67(6), 2522 (1994).

${ }^{22}$ C. E. Chan and D. J. Odde, Science 322(5908), 1687 (2008).

${ }^{23}$ R. P. McEver and C. Zhu, Annu. Rev. Cell Dev. Biol. 26, 363 (2010).

${ }^{24}$ K. C. Chang and D. A. Hammer, Biophys. J. 76(3), 1280 (1999).

${ }^{25}$ L. B. Freund, Proc. Natl. Acad. Sci. U.S.A. 106(22), 8818 (2009).

${ }^{26}$ F. Kong, A. J. Garcia, A. P. Mould, M. J. Humphries, and C. Zhu, J. Cell Biol. 185(7), 1275 (2009).

${ }^{27}$ W. Chen, J. Z. Lou, E. A. Evans, and C. Zhu, J. Cell Biol. 199(3), 497 (2012).

${ }^{28}$ E. A. Evans and D. A. Calderwood, Science 316(5828), 1148 (2007).

${ }^{29}$ M. Arnold, E. A. Cavalcanti-Adam, R. Glass, J. Blummel, W. Eck, M. Kantlehner, H. Kessler, and J. P. Spatz, ChemPhysChem 5(3), 383 (2004).

${ }^{30}$ D. Leckband and J. Israelachvili, Q. Rev. Biophys. 34(2), 105 (2001).

${ }^{31}$ H. J. Gao, J. Qian, and B. Chen, J. R. Soc., Interface 8(62), 1217 (2011).

${ }^{32}$ The Monte Carlo simulations were performed by letting single receptors randomly hop in the truncated harmonic potential, and make a bond at rate $k_{\mathrm{on}}^{0}$ if a receptor falls into the binding distance near the opposing ligand. The initial position of receptors was set to be zero and the total elapsed time until successful bond making was recoded as "waiting time."

${ }^{33}$ T. Erdmann and U. S. Schwarz, Biophys. J. 91(6), L60 (2006).

${ }^{34}$ D. A. Lauffenburger and J. J. Linderman, Receptors: Models for Binding, Trafficking, and Signalling (Oxford University Press, New York, 1993).

${ }^{35}$ M. L. Dustin and D. Depoil, Nat. Rev. Immun. 11(10), 672 (2011). 